This item was submitted to Loughborough's Research Repository by the author.

Items in Figshare are protected by copyright, with all rights reserved, unless otherwise indicated.

\title{
Effect of air-jet texturing on adhesion behaviour of technical polyester yarns to rubber
}

PLEASE CITE THE PUBLISHED VERSION

http://dx.doi.org/10.1163/016942410X549997

\section{PUBLISHER}

(c) Koninklijke Brill NV, Leiden, published by Taylor and Francis

\section{VERSION}

AM (Accepted Manuscript)

\section{PUBLISHER STATEMENT}

This work is made available according to the conditions of the Creative Commons Attribution-NonCommercialNoDerivatives 4.0 International (CC BY-NC-ND 4.0) licence. Full details of this licence are available at: https://creativecommons.org/licenses/by-nc-nd/4.0/

\section{LICENCE}

CC BY-NC-ND 4.0

\section{REPOSITORY RECORD}

Koc, Serpil Koral, Asli Sengonul Hockenberger, and Memis Acar. 2019. "Effect of Air-jet Texturing on Adhesion Behaviour of Technical Polyester Yarns to Rubber". figshare. https://hdl.handle.net/2134/19366. 


\title{
Effect of Air-Jet Texturing on Adhesion Behaviour of Technical Polyester Yarns to Rubber
}

\author{
Serpil Koral Koc, Asli Sengonul Hockenberger \\ Uludag University, Faculty of Engineering and Architecture, Textile Engineering Department, 16059 Gorukle-Bursa, \\ Turkey \\ Memis Acar \\ Wolfson School of Mechanical and Manufacturing Engineering Loughborough University, Leicestershire LE11 3TU, \\ UK
}

\begin{abstract}
Air-jet texturing of technical polyester yarns was performed in order to improve its adhesion to rubber. The air-jet texturing parameters were selected with great care to minimize the mechanical loss. $\mathrm{H}$-adhesion tests were used to characterize the adhesion of the yarns to rubber. A significant increase in the adhesion of dimensionally stable polyethylene terephthalate yarn, textured with an overfeed level of 15\% (DSPET15), was recorded, while a decrease in the adhesion of high tenacity polyethylene terephthalate (HTPET) yarn was observed for all overfeed levels. The effects of air-jet texturing on the adhesion of technical polyester yarns were discussed in terms of changes in the yarn geometry and changes on the single fiber surfaces. Changes in the yarn geometry were investigated by optical microscopy studies, while changes on the fiber surface were investigated by scanning electron microscopy (SEM), atomic force microscopy (AFM) and environmental scanning electron microscopy (ESEM) studies. It was observed that air-jet texturing alters both the yarn geometry and the single fiber surfaces, leading to a change in the adhesion to rubber.
\end{abstract}

Keywords

Technical polyester yarn, air-jet texturing, fiber reinforced rubber composite, adhesion

\section{Introduction}

Textile fibers combine low density with exceptional mechanical properties and as a result have found increasing usage as reinforcing elements in fiber-reinforced composites [1]. Polyester fibers have superior mechanical and thermal properties and also show good resistance to chemicals [2]. Due to these desirable properties there is an increasing demand for the use of polyester fibers in composite materials. Besides polyethylene terephthalate (PET), different polyesters such as polyethylene naphthalate (PEN) and Vectran are also used in industrial applications. Composites based on fiber and rubber have the widest application. The mechanical properties of fiber-reinforced composites depend not only on the properties of fibers and matrices, but also on the nature of the fiber surface and the mechanism of load transfer from the fibers to the matrix at the interface [1]. Adhesion is one of the most important surface properties of these materials and can be defined as the mechanical resistance to separation of a system of bonded materials [3]. However, development of adequate adhesion between fibers and rubber is a challenge since there are significant differences between synthetic fibers and elastomer rubber from both a chemical as well as from a mechanical point of view. In the most recent applications the adhesive system, resorcinol-formaldehyde-latex (RFL) has been used. However the normal RFL treatment does not provide adequate adhesion for polyester owing to the hydrophobic nature of its surface [4]. Various methods have been proposed for improving the adhesion of polyester fibers to rubber $[1,2,4]$. However, most of these techniques are either very expensive, difficult or not very environmentally friendly.

Air-jet texturing is one of the most popular texturing methods. Unlike other texturing methods it is a fully mechanical process using a turbulent fluid, which is usually compressed air. Loops are formed on the surface of the filament yarn, giving it a voluminous character [5-7]. 
In this study air-jet texturing of technical polyester yarns was performed in order to improve their adhesion to rubber. The effects of the air-jet texturing process on both the yarns and single fibers were investigated and the relation between air-jet texturing process and the adhesion of technical polyesters to rubber was discussed.

\section{Experimental}

\subsection{Materials}

In this study the following four different technical polyester yarns were used: dimensionally stable PET (DSPET); high tenacity PET (HTPET); PEN and Vectran. Conventional PET (FDYPET) was also used in order to make a comparison with the technical yarns. All the yarns were commercially available and were obtained from the producer companies. Properties of the yarns are given in Table 1.

The resorcinol-formaldehyde-latex (RFL) solution and the rubber compound used for the adhesion tests were kindly provided by KordSA Global (Turkey).

\subsection{Air-Jet Texturing Studies}

It is well known that in air-jet texturing, feeder yarn properties and texturing process parameters influence the structure and properties of the textured yarns [8]. It is also well known that air-jet texturing results in a mechanical loss of yarn strength owing to the disorientation of the single filaments and fiber damage. The yarns in this study are expected to be used in technical applications where mechanical properties are crucial. Therefore air-jet texturing parameters were selected with great care in order to obtain a homogeneous yarn structure with minimum strength loss. To obtain these desired properties the yarns were fed to the machine in a single end method with low overfeed levels. The FDYPET yarn which has a lower linear density was fed into the machine with three ends to keep the linear density of the supply yarns similar and to obtain comparable results. The feed yarns were subjected to drawing between heated feed rollers to improve their mechanical properties. The draw ratio was selected to comply with the limitations of the texturing machine. Each of the yarns used in this study had a different temperature-dependent behaviour; as a result the temperature of the feed rollers was adjusted to $15^{\circ} \mathrm{C}$ above the glass transition temperature of each yarn in order to obtain comparable effects. In the case of Vectran, the temperature of the feed rollers was set at maximum since it has no clear glass transition temperature.

The air-jet texturing studies were carried out on a SSM Stähle RM3T machine, with the following parameters: $0.8 \mathrm{MPa}$ air pressure, $300 \mathrm{~m} / \mathrm{min}$ texturing speed, $210^{\circ} \mathrm{C}$ heat-setting temperature, Hemajet A357 type of nozzle, 3\% mechanical stretch, 3 different overfeed levels; 10, 15 and $20 \%$. The given yarn codes and the production parameters are given in Table 2.

\subsection{Tensile Measurements}

Tensile measurements were performed on a 4301 Instron tensile tester with a crosshead speed of 300 $\mathrm{mm} / \mathrm{min}$ and a gauge length of $500 \mathrm{~mm}$ (ASTM D 2256).

\subsection{Optical Microscopic Studies}

The filament arrangement within the yarn and the yarn ends pulled out from the rubber compound in the $\mathrm{H}$-adhesion tests were investigated using an Olympus SZ6045 Model Automatic Trinoculer Stereo Zoom Microscope. Air-jet texturing studies, tensile measurements and optical microscopic studies were performed in the Laboratories of Uludag University, Textile Engineering Department (Bursa, Turkey).

\subsection{Scanning Electron Microscopy (SEM)}

The scanning electron microscopy studies were carried out using a Jeol JSM6335F model scanning electron microscope in the Laboratories of the Scientific and Technological Research Council of Turkey, Marmara Research Center (Kocaeli, Turkey). These studies analysed the surface topography of the single fibers before and after air-jet texturing. All the specimens were sputter-coated with gold and analysed at $10 \mathrm{kV}$. 
Table 1. Yarn properties and the given yarn codes

\begin{tabular}{lllllll}
\hline $\begin{array}{l}\text { Chemical structure } \\
\text { and type of the } \\
\text { filaments }\end{array}$ & Producer & $\begin{array}{l}\text { Yarn } \\
\text { code }\end{array}$ & $\begin{array}{l}\text { Yarn } \\
\text { count } \\
\text { (tex) }\end{array}$ & $\begin{array}{l}\text { Number of } \\
\text { filaments }\end{array}$ & $\begin{array}{l}\text { Glass transition } \\
\text { temperature } \\
\left({ }^{\circ} \mathrm{C}\right)\end{array}$ & $\begin{array}{l}\text { Melting } \\
\text { point } \\
\left({ }^{\circ} \mathrm{C}\right)\end{array}$ \\
\hline $\begin{array}{l}\text { Conventional PET } \\
\text { (fully drawn) }\end{array}$ & Korteks & FDYPET & 33.50 & 72 & 69 & 252.3 \\
$\begin{array}{l}\text { Dimensionally } \\
\text { stable PET }\end{array}$ & $\begin{array}{l}\text { Performance } \\
\text { fibers }\end{array}$ & DSPET & 110 & 300 & 73 & 254.1 \\
$\begin{array}{l}\text { High tenacity PET } \\
\text { Performance } \\
\text { fibers }\end{array}$ & HTPET & 110 & 192 & 123 & 255.5 \\
PEN & $\begin{array}{l}\text { Performance } \\
\text { fibers }\end{array}$ & PEN & 110 & 200 & 76 & 272.2 \\
Kectran & Kuraray & Vectran & 110 & 200 & - & 323.5 \\
\hline
\end{tabular}

Table 2. Details of the air-jet texturing process

\begin{tabular}{llllc}
\hline $\begin{array}{l}\text { Type of the } \\
\text { feed yarn }\end{array}$ & $\begin{array}{l}\text { Yarn } \\
\text { code }\end{array}$ & $\begin{array}{l}\text { Feed yarns } \\
\text { used }\end{array}$ & $\begin{array}{l}\text { Overfeed } \\
(\%)\end{array}$ & $\begin{array}{l}\text { Temperature of the } \\
\text { feed rollers }\left({ }^{\circ} \mathrm{C}\right)\end{array}$ \\
\hline FDYPET & FDY10 & 3 cores & 10 & 84 \\
& FDY15 & & 15 & \\
& FDY20 & & 20 & \\
DSPET & DSPET10 & 1 core & 10 & 88 \\
& DSPET15 & & 15 & \\
& DSPET20 & & 20 & \\
HTPET & HTPET10 & 1 core & 10 & 138 \\
& HTPET15 & & 15 & \\
& HTPET20 & & 20 & \\
PEN & PEN10 & 1 core & 10 & 91 \\
& PEN15 & & 15 & \\
& PEN20 & & 20 & \\
Vectran & Vectran10 & 1 core & 10 & 200 \\
& Vectran15 & & 15 & \\
& Vectran20 & & 20 & \\
\hline
\end{tabular}

\subsection{Atomic Force Microscopy (AFM)}

Atomic force microscopy (AFM) is a powerful tool used to analyse the surface characteristics of textile fibres since it allows one to investigate textile fibres without any coating and at ambient conditions. In this study AFM studies were performed on a Benyuan CSPM4000 model atomic force microscope, in the Laboratories of Jiangnan University (Wuxi, China). The fibers were immobilized on the magnetic atomic force microscope sample stubs with the help of double-sided tape. The scannings were carried out in tapping mode using silicon cantilevers with a nominal spring constant of $42 \mathrm{~N} / \mathrm{m}$.

\subsection{Environmental Scanning Electron Microscopy (ESEM)}

Environmental scanning electron microscopy was developed in the mid eighties. Its primary advantage lies in permitting the microscopist to vary the sample environment through a range of pressures, temperatures and gas compositions. ESEM offers high resolution secondary electron imaging in a gaseous environment of practically any composition, at pressures as high as 50 Torr, and temperatures as high as $1500^{\circ} \mathrm{C}$. Moreover wet, oily, dirty, non-conductive samples may be examined in their natural state without modification or preparation [9]. Dynamic experiments can also 
be performed with ESEM in wet mode. In this mode, as the relative humidity reaches $100 \%$, water condenses on to the surface of the sample. By observing the water droplets, wetting behaviour of samples can be investigated [10].

ESEM studies have been carried out using a Philips XL30 ESEM-FEG environmental scanning electron microscope, in wet mode, with a Peltier cooling stage in the Laboratories of Illinois University (Urbana, IL, USA), with an acceleration voltage of $20 \mathrm{kV}$.

\subsection{H-Adhesion Tests}

The adhesion strength between yarn and rubber was evaluated using an $\mathrm{H}$-adhesion test. In order to achieve the adhesion tests, firstly the yarns was treated with an adhesive system (RFL solution) by using a commercial dip-stretch cord processing machine (Litzler Computreater 2000), which consists of a typical RFL bath [11] followed by a heat treatment zone. The dipping process was performed using single dip method in order to observe the effects of air-jet texturing process on the adhesion of polyester yarns on rubber.

After the dipping process, the coated yarns were embedded in rubber and cured at $153^{\circ} \mathrm{C}$, for $25 \mathrm{~min}$ at a pressure of 3.2 MPa. Then the products were cut into H-shaped samples. The pull-out forces of the samples were measured using an Instron 4502 at a crosshead speed of $300 \mathrm{~mm} / \mathrm{min}$, ASTM D4776.

\section{Results and Discussion}

Table 3 shows the tensile results of the yarns before and after air-jet texturing. The results show a reduction in tenacity for all the yarns after air-jet texturing except for FDY10 and FDY15. The amount of reduction increases with the increase of the overfeed level. The air-jet texturing process disrupts, entangles and changes the parallel arrangement of the filaments and creates surface loops anchored in the yarn core [6]. This alteration of the yarn structure affects the mechanical properties of the final yarn. The disturbance of the filaments leads to a decrease in both tenacity and breaking extension of the textured yarns when compared to their feeder yarns [8]. However, increased overfeed level cause an increase in breaking extension due to opening up of the looped structure. The reason for the different behaviour of FDYPET yarns can be understood by analysing the stressstrain curves of the single fibers (Fig. 1). Stress-strain curve of FDYPET fiber shows a clear cold drawing region after the yield [12]. This indicates that, in the amorphous region of FDYPET fiber, there are some domains which can be more easily orientated. Other fibers don't show such a region. Therefore, to perform air-jet texturing on all the samples under the same conditions, and to improve their mechanical properties to minimize the strength losses due to air-jet texturing, partial orientation was obtained by an initial drawing of all samples. This partial orientation resulted in an increase in the tenacity of FDYPET yarns.

Table 4 shows the results of the $\mathrm{H}$-adhesion tests. The results show an improvement in adhesion for all the technical polyester fibers to rubber, except for FDY10, Vectran10 and the HTPET group, after air-jet texturing. The most significant improvement was seen in the DSPET yarns. In particular, the adhesion force for DSPET15 increased by about 344\% compared to the adhesion force before texturing. Generally the maximum increment was obtained for yarns textured with an overfeed level of $15 \%$. On the other hand a slight decrease in adhesion force for both FDY10 and Vectran10 was observed. An interesting result is the decrease in adhesion force of HTPET yarns for all the overfeed levels. Although HTPET and DSPET yarns have the same chemical structure, the amorphous orientation of HTPET fibers is higher than in the DSPET fibers [13]. Higher amorphous orientation causes lower-dimensional stability leading to a reduction of the integrity of the filament bundle within the rubber compound. This is thought to be the main reason for the reduction in the adhesion to rubber in the case of HTPET yarns. 
Table 3. Tensile results of the yarns before and after air-jet texturing

\begin{tabular}{cclr}
\hline Yarn code & $\begin{array}{l}\text { Yarn count } \\
\text { (tex) }\end{array}$ & $\begin{array}{l}\text { Tenacity } \\
\text { (N/tex) }\end{array}$ & $\begin{array}{l}\text { Breaking extension } \\
(\%)\end{array}$ \\
\hline FDYPET Raw & 33.50 & 0.332 & 32.90 \\
FDY10 & 83.28 & 0.457 & 8.22 \\
FDY15 & 86.83 & 0.354 & 8.15 \\
FDY20 & 89.95 & 0.286 & 8.37 \\
DSPET Raw & 110.00 & 0.600 & 12.92 \\
DSPET10 & 115.81 & 0.405 & 8.74 \\
DSPET15 & 119.97 & 0.399 & 9.10 \\
DSPET20 & 124.49 & 0.325 & 9.24 \\
HTPET Raw & 110.00 & 0.711 & 13.83 \\
HTPET10 & 115.54 & 0.557 & 11.40 \\
HTPET15 & 121.34 & 0.498 & 12.53 \\
HTPET20 & 121.83 & 0.414 & 12.47 \\
PEN Raw & 110.00 & 0.733 & 15.88 \\
PEN10 & 124.34 & 0.409 & 14.98 \\
PEN15 & 125.87 & 0.324 & 17.06 \\
PEN20 & 128.28 & 0.308 & 20.99 \\
Vectran Raw & 110.00 & 2.239 & 6.19 \\
Vectran10 & 116.47 & 0.953 & 6.88 \\
Vectran15 & 119.42 & 0.883 & 11.17 \\
Vectran20 & 122.05 & 0.814 & 13.59 \\
\hline
\end{tabular}

The adhesion between the fiber and rubber is a combination of three bonding systems; mechanical; chemical; and molecular bonding [4]. Air-jet texturing is only a mechanical process and therefore no change in the chemical and molecular bonding is expected. Moreover it is known that the bulky yarn structure obtained after texturing does not cause an increase in the dip penetration depth [14]. For these reasons the change in the adhesion behaviour of the yarns was attributed to changes on the fiber surface and in the yarn geometry.

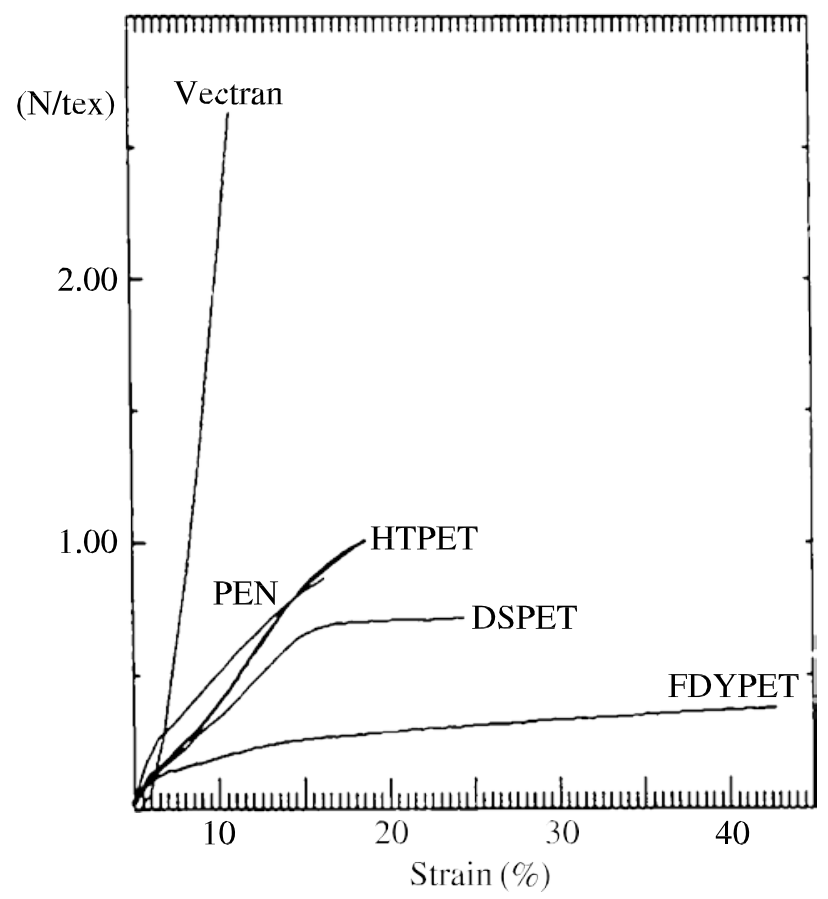

Figure 1. Stress-strain curves of single fibers. 
Optical microscopy images show the entanglements and the looped structure of the air-jet textured yarns (Fig. 2). The images also show that the amount of the entanglement and the type of the loops, vary, depending on the feed yarn [12].

The changes in the surface of single fibers were investigated using SEM, AFM and ESEM analyses. In most cases the AFM analysis gave a more detailed image than did the SEM analysis. However we believe that it is beneficial to perform both an AFM and an SEM analysis.

Table 4. H-adhesion test results

\begin{tabular}{cl}
\hline Yarn code & Adhesion force $(\mathrm{N})$ \\
\hline FDYPET Raw & $26.72 \pm 2.52$ \\
FDY10 & $26.04 \pm 3.18$ \\
FDY15 & $39.84 \pm 3.26$ \\
FDY20 & $36.30 \pm 3.93$ \\
DSPET Raw & $32.42 \pm 3.63$ \\
DSPET10 & $34.73 \pm 4.20$ \\
DSPET15 & $111.69 \pm 5.42$ \\
DSPET20 & $101.45 \pm 5.93$ \\
HTPET Raw & $95.96 \pm 5.49$ \\
HTPET10 & $28.33 \pm 3.31$ \\
HTPET15 & $34.30 \pm 4.61$ \\
HTPET20 & $36.08 \pm 4.35$ \\
PEN Raw & $15.25 \pm 2.15$ \\
PEN10 & $28.85 \pm 2.62$ \\
PEN15 & $35.93 \pm 4.95$ \\
PEN20 & $35.32 \pm 4.33$ \\
Vectran Raw & $28.89 \pm 3.52$ \\
Vectran10 & $21.83 \pm 2.67$ \\
Vectran15 & $45.28 \pm 4.42$ \\
Vectran20 & $41.35 \pm 4.97$ \\
\hline
\end{tabular}

In order to predict the adhesion potential of fibers to a matrix (which they strengthen) it is useful to know the wettability of fibers [15]. However, direct measurement of wettability of a single fiber is difficult due to its small diameter. In this study ESEM was used to analyse the wettability of the samples before and after air-jet texturing. From ESEM studies it is possible to get an idea of the wettability of a sample by observing the shape and number of the droplets on its surface. The wettability of fibers is influenced by the chemical structure, production parameters such as drawing, heat setting and the application of a spin finish etc. The samples used in this study were all polyester fibers; however they all have very different production parameters. Moreover, PEN and Vectran have different chemical structures. Therefore they showed different wetting behaviours before air-jet texturing. Fiber type is one of the most important parameters in air-jet texturing. Consequently, airjet texturing had different effects on different fibers. As a result, the samples showed different wetting properties, both before and after texturing. 

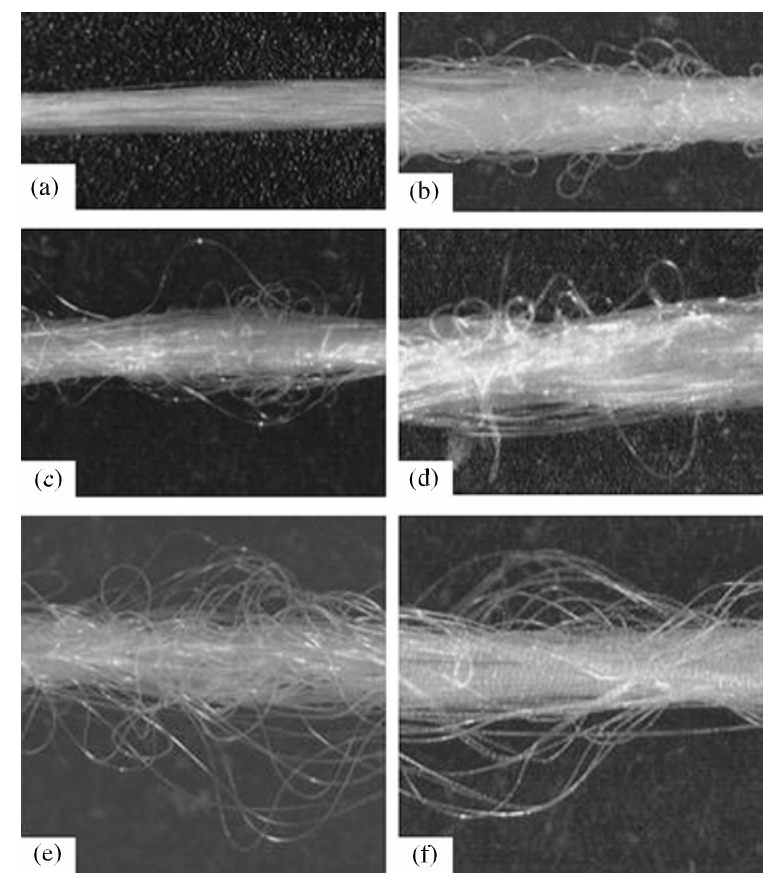

Figure 2. Optical microscopy images of the air-jet textured yarns: (a) a typical polyester yarn before air-jet texturing, (b) FDY20, (c) DSPET20, (d) HTPET20, (e) PEN20 and (f) Vectran20.

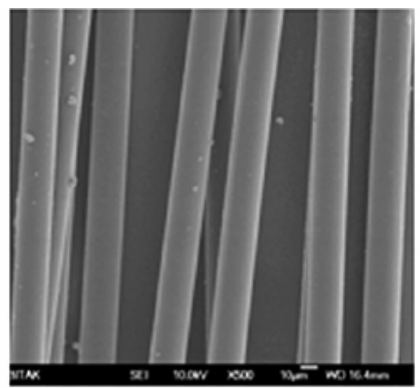

(a)

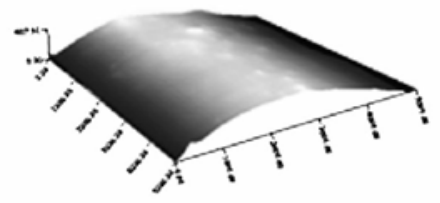

(c)

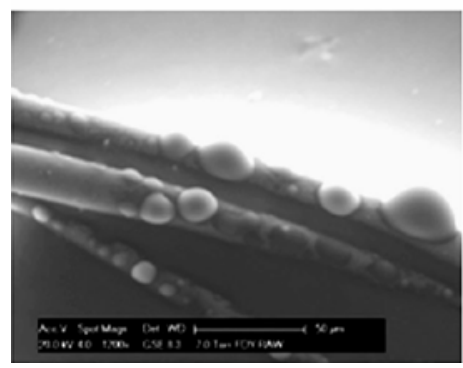

(e)

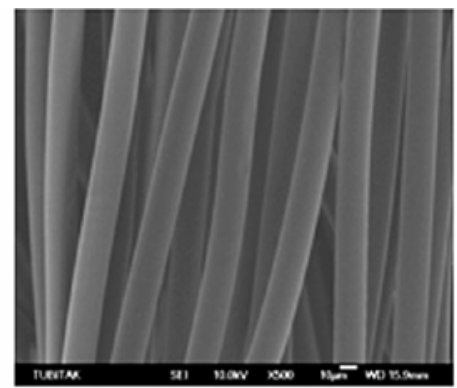

(b)

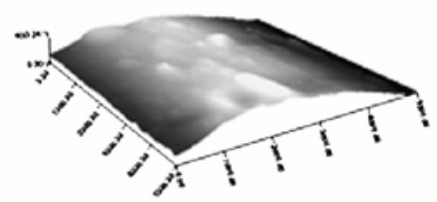

(d)

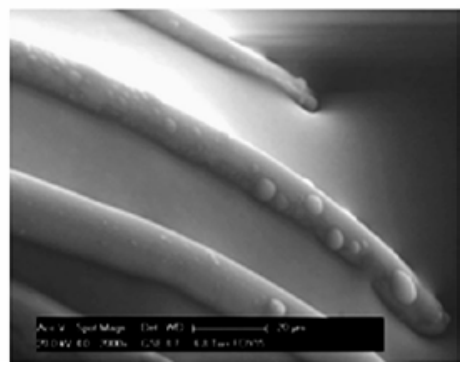

(f)

Figure 3. SEM, AFM and ESEM images of FDYPET fibers respectively: (a) FDYPET Raw, (b) FDY20, (c) FDYPET Raw, (d) FDY15, (e) FDYPET Raw and (f) FDY15. 
Figure 3 shows the SEM, AFM and ESEM images of FDYPET fibers. Before texturing the FDYPET fibers have a smooth surface with some impurities. After texturing the surfaces were clear and smooth with no pronounced differences. Texturing had removed the impurities and the spin finish, from the surfaces. However AFM image of the yarn with the 15\% overfeed level, shows some kinks on the surface together with spin finish residue (Fig. 3(d)). The water droplets in the ESEM images of the FDYPET fibers show spherical cap shapes with a high contact angle indicating the hydrophobic property of the fibres [10]. After texturing, the water droplets show flattened cap shapes, indicating an improvement in the wetting behaviour of FDTPET fibers due to the removal of the spin finish. The changes in the shape of the water droplets are most significant in the ESEM image of the FDY15 fibers (Fig. 3(f)).

The SEM images of DSPET fibers show that DSPET filaments have a smooth surface with some spin finish and impurities (Fig. 4(a)). This can also be seen from the AFM images. After texturing, fibrillation and peeling off of the surface at some places was observed. Fibrillation on the filament surface is clearly visible, especially in the AFM image of DSPET10 (Fig. 4(d)). Before texturing, many small, flat water droplets were observed in the ESEM images of the DSPET fibers. After texturing no significant change to the shape of the droplets was observed. However the number of the droplets decreased markedly due to the surface changes (Fig. 4(f)).

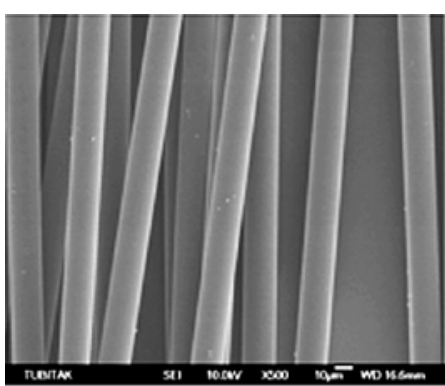

(a)

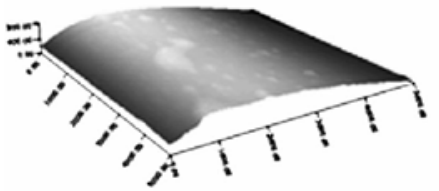

(c)

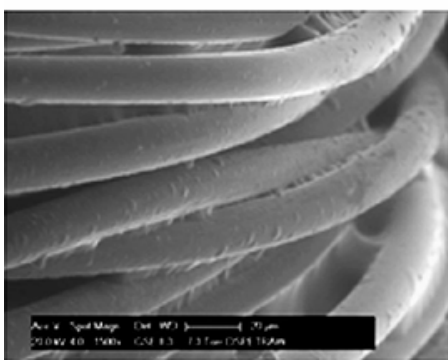

(e)

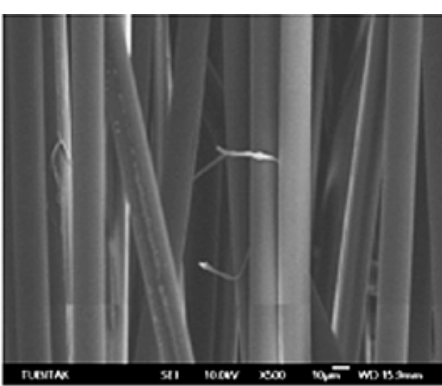

(b)

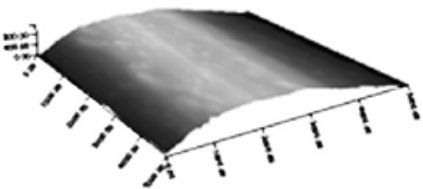

(d)

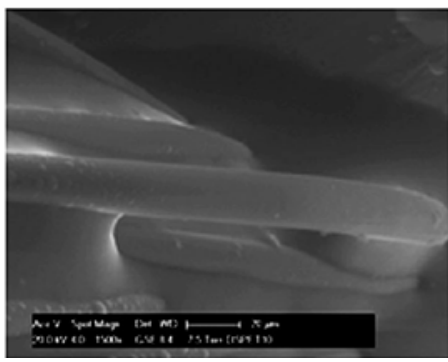

(f)

Figure 4. SEM, AFM and ESEM images of DSPET fibers respectively: (a) DSPET Raw, (b) DSPET20, (c) DSPET Raw, (d) DSPET10, (e) DSPET Raw and (f) DSPET15.

The SEM images of HTPET filaments show even and smooth surfaces with some very small impurities and spin finish before texturing (Fig. 5). However after texturing, a peeling off of the surface was observed. The AFM images also confirm these results. The HTPET fiber surfaces show very few water droplets as seen in Fig. 5(e). However it was observed that many water droplets were formed on the surfaces of the HTPET fibers after texturing (Fig. 5(f)). 


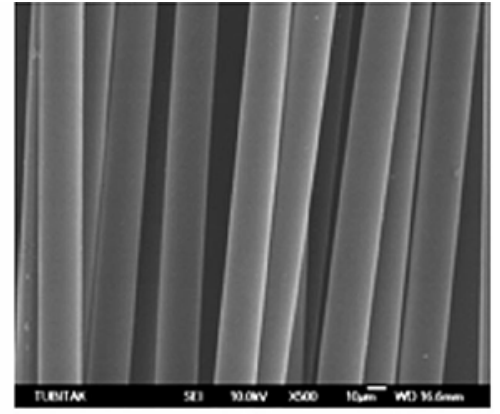

(a)

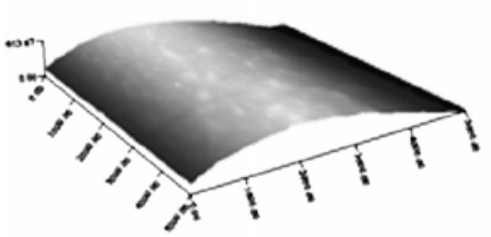

(c)

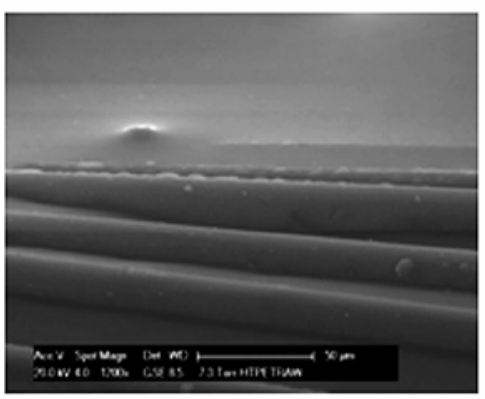

(e)

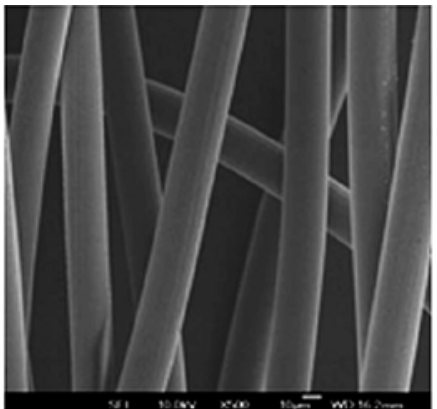

(b)

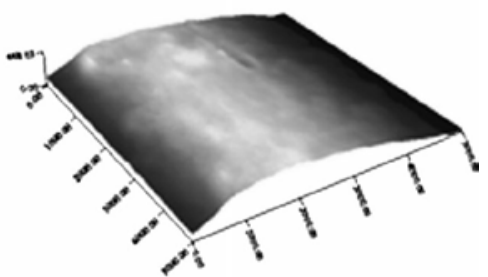

(d)

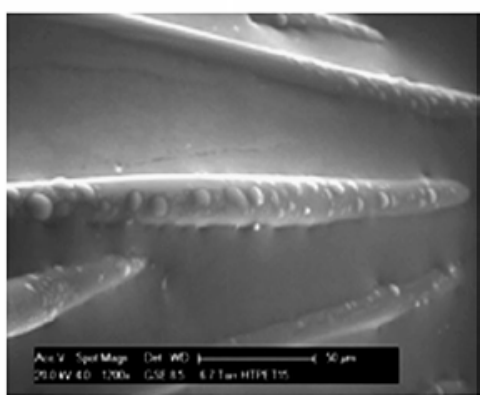

(f)

Figure 5. SEM, AFM and ESEM images of HTPET fibers respectively: (a) HTPET Raw, (b) HTPET20, (c) HTPET Raw, (d) HTPET20, (e) HTPET Raw and (f) HTPET15.

Before texturing many impurities were observed on the surface of the PEN fibers (Fig. 6(a)). After texturing almost all the impurities were removed from the fiber surfaces and the fiber surfaces appeared smooth. However the AFM images showed a rougher surface after texturing. Therefore it can be concluded that air-jet texturing can cause changes on the surface at the nano level which can only be seen with AFM studies (Fig. 6(d)). The PEN fibre surfaces showed large spherical cap shapes before texturing. After texturing the most significant change was observed on the surface of PEN15 fibers. The size of water droplets had reduced in size and their shape were flattened as a result of the increase in roughness after texturing (Fig. 6(f)).

Vectran fibers show fibrillated surfaces with some defects before texturing. These defects can be attributed to the high modulus of Vectran fibers as well as to their anisotropic structure. After texturing, the amount of fibrillation increased. Moreover some kink bands were also observed due to buckling and breaking of the stiff polymer chains [16] (Fig. 7(c)). The AFM images gives a more detailed description of the surface topography of the Vectran fibers. Kink bands on the surface of 
Vectran20 can easily be recognised from the AFM images (Fig. 7(e)). In the ESEM images of the Vectran fibers, a few relatively flat droplets were observed. After texturing the number of the droplets increased. However no pronounced change was noticed in the shapes of the droplets (Fig. 7(g)).

Images obtained from SEM, AFM and ESEM analysis indicate that air-jet texturing caused some changes on the fiber surface, leading to changes in the wetting behaviour of the fibers, depending on the fiber type.

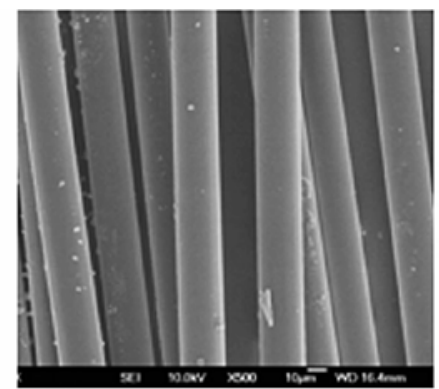

(a)

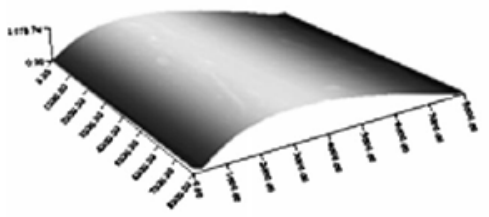

(c)

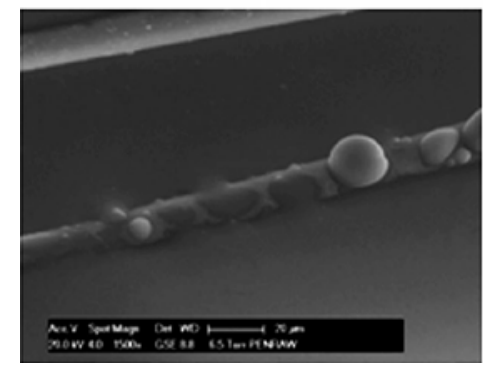

(e)

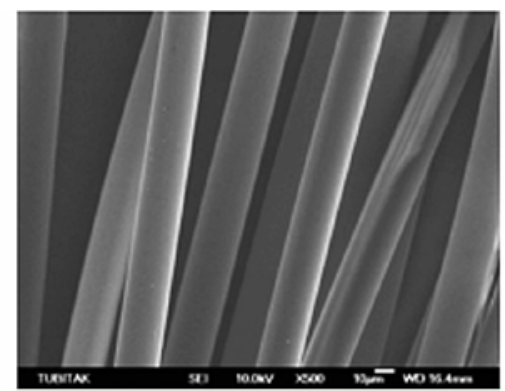

(b)

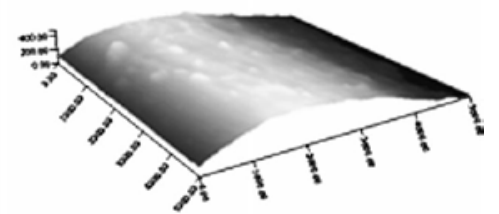

(d)

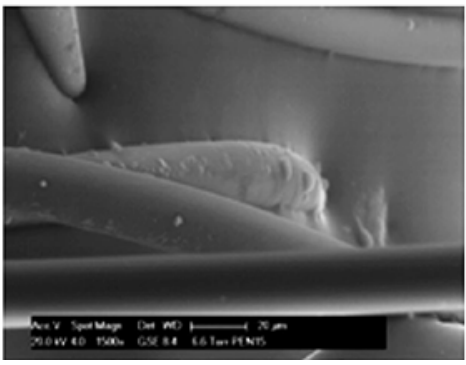

(f)

Figure 6. SEM, AFM and ESEM images of PEN fibers respectively: (a) PEN Raw, (b) PEN20, PEN Raw, (d) PEN20, (e) PEN Raw and (f) PEN15.

The optical microscopy images of the yarns after the dipping process are given in Fig. 8. The images show that after dipping the size of the loops become smaller but the overall looped yarn structure did not change. This indicates that the dipping process did not remove the air-jet texturing effect. Another point is that the RFL does not cluster around the loops. It only covers the fiber surfaces.

The yarn ends, pulled out of the rubber after the H-adhesion tests, was also investigated by optical microscope since it gives information about the strength of the interface. The images show that there are small rubber residues on the surface of the pulled out ends before texturing. However, after texturing the amount of the rubber residues increased indicating an increase in the adhesion between the rubber and the filaments. It was also observed that the integrity of the filament bundle was significantly disturbed in the HTPET15 and PEN15, while no significant change was observed in the other samples (Fig. 9). 


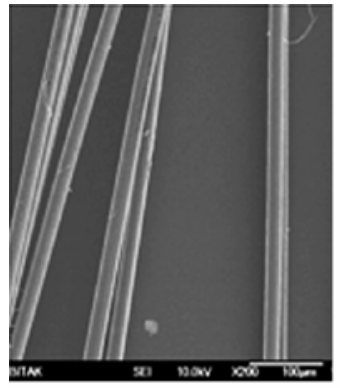

(a)

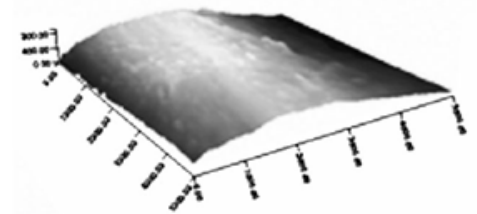

(d)

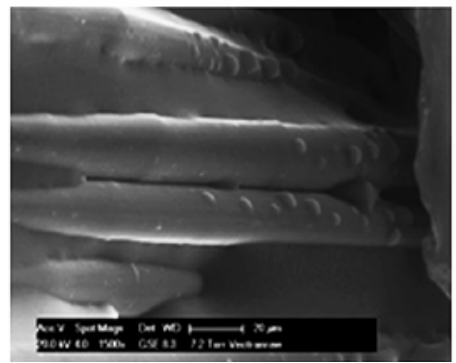

(f)

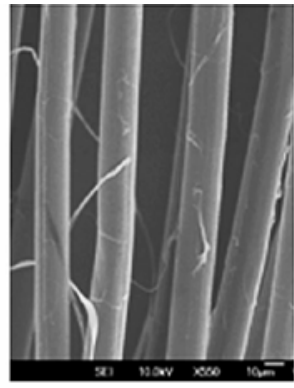

(b)

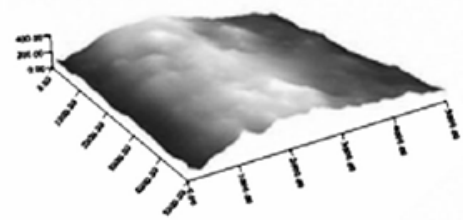

(e)

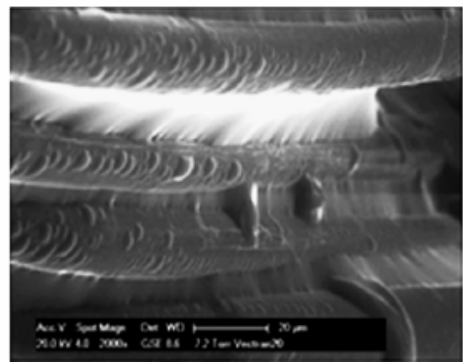

(g)

Figure 7. SEM, AFM and ESEM images of Vectran fibers respectively: (a) Vectran Raw, (b) Vectran20, (c) Vectran20, (d) Vectran Raw, (e) Vectran 20, (f) Vectran Raw and (g) Vectran15.
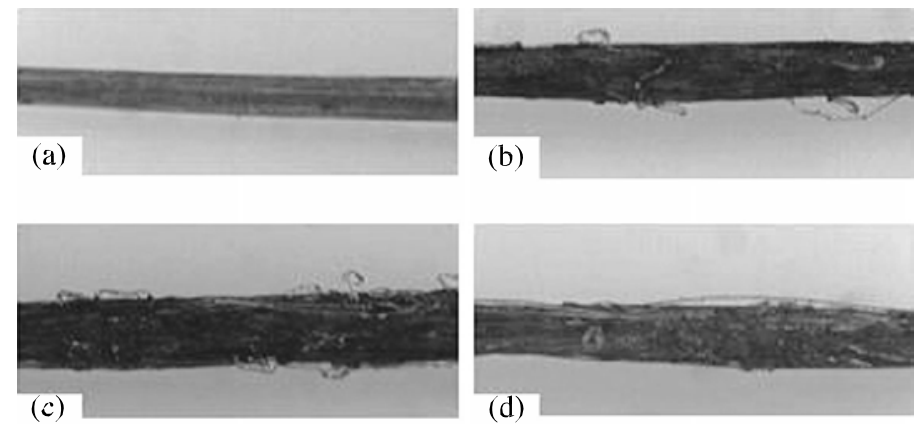

(c)

(d)
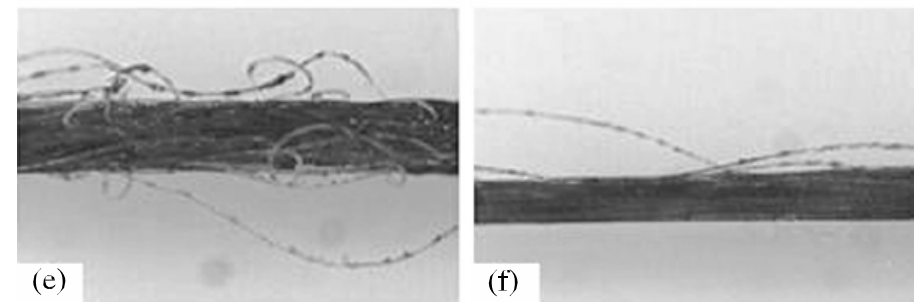

Figure 8. Optical microscopy images of the air-jet textured yarns after dipping process: (a) a typical polyester yarn before air-jet texturing, (b) FDY20, (c) DSPET20, (d) HTPET20, (e) PEN20 and (f) Vectran20. 


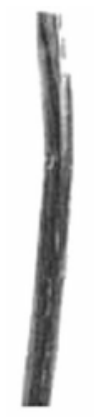

(a)

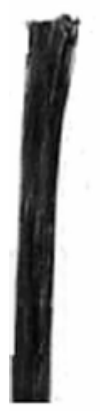

(e)

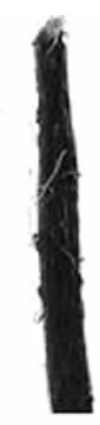

(b)

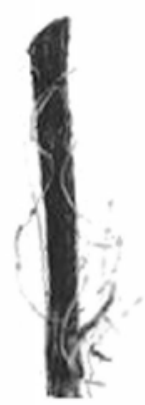

(f)

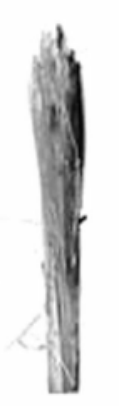

(c)

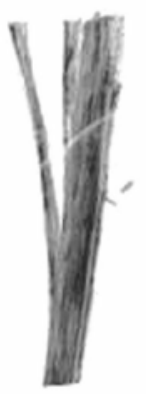

(g)

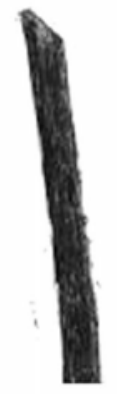

(d)

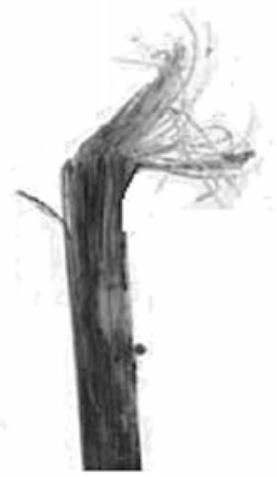

(i)

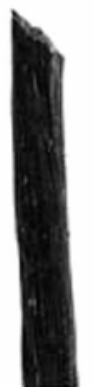

(j)

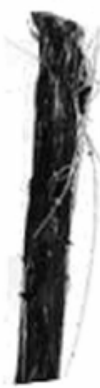

(h)

Figure 9. Optical microscopy images of the yarn ends pulled out the rubber: (a) FDYPET Raw, (b) FDY15, (c) DSPET Raw, (d) DSPET15, (e) HTPET Raw, (f) HTPET15, (g) PEN Raw, (h) PEN15, Vectran Raw and (j) Vectran15.

\section{Conclusions}

Air-jet texturing alters both the feeder yarn geometry and single fiber surfaces. It disrupts the parallel arrangement of the filaments and creates surface loops anchored in the yarn core. It also causes fibrillation, peeling off and kink bands on the fiber surfaces and removes impurities. The change in the yarn geometry and fiber surfaces resulted in a reduction in the tenacity of the feeder yarns. However in this study owing to the initial drawing of the feed yarns an increase in the tenacity values of FDY10 and FDY15 was observed.

Air-jet texturing produces bulkier yarns. However it was observed that this bulky structure does not contribute to an increase in dip penetration depth [14]. Therefore the increase in the adhesion of the technical polyesters was mainly attributed to the increased surface area due to the looped yarn and fibrillated, peeled off and rougher fiber surface structure.

In this work we have shown that air-jet texturing improves the adhesion of technical polyester yarns (apart from HTPET yarns) to rubber. This improvement is particularly noticeable for DSPET 
yarns. The HTPET and DSPET yarns have the same chemical structure, but have different amorphous orientations. The amorphous orientation of HTPET fibers is higher than that of the DSPET fibers [13]. Higher amorphous orientation causes lower-dimensional stability, which is thought to be the main reason for the breakdown of the integrity of the filament bundles. This, we believe, caused the decrease in the adhesion between the rubber and the HTPET yarns.

\section{Acknowledgements}

The authors would like to thank KordSA Global, Turkey; Korteks, Turkey, for their support and valuable information and Prof. Qufu Wei of Jiangnan University, China for the AFM studies. This work was supported by The Scientific and Technological Research Council of Turkey, the project number is $106 \mathrm{M} 131$.

\section{References}

1. S. Luo and W. J. Ooij, J. Adhes. Sci. Technol. 16, 1715 (2002).

2. Y. Hsieh, in: Surface Characteristics of Fibers and Textiles, C. M. Pastore and P. Kiekens (Eds), pp. 33-57. Markel Dekker Inc., USA (2001).

3. E. M. Liston, L. Martinu and M. R. Wertheimer, in: Plasma Surface Modification of Polymers: Relevance to Adhesion, M. Strobel, C. S. Lyons and K. L. Mittal (Eds), pp. 3-42. VSP, Netherlands (1994).

4. S. K. Chawla, in: Synthetic Fibre Materials, H. Brody (Ed.), pp. 203-237. Longman Scientific and Technical, Essex, England (1994).

5. M. Acar, An analysis of the air-jet yarn texturing process and the development of improved nozzles, $P h D$ Thesis, Loughborough University of Technology (1987).

6. A. Demir and H. M. Behery, Synthetic Filament Yarn Texturing Technology. Prentice Hall, New Jersey (1997).

7. J. W. S. Hearle, L. Hollick and D. K. Wilson, Yarn Texturing Technology. Woodhead Publishing, Cambridge (2001).

8. R. S. Rengasamy, V. K. Kothari and A. Patnaik, Textile Res. J. 74, 259 (2004).

9. www.calce.umd.edu/general/Facilities/ESEM.pdf, short overview about the ESEM, K. Kimseng and M. Meissel, 04.01.2010.

10. Q. Wei, X. Q. Wang, R. R. Mather and A. F. Fotheringham, Fibres Text. East. Eur. 12, 79 (2004).

11. M. Jamshidi, F. Afshar, N. Mohammadi and S. Pourmahdian, Appl. Surf. Sci. 249, 208 (2005).

12. S. K. Koc and A. S. Hockenberger, Tekstil ve Konfeksiyon 4, 299 (2010).

13. P. B. Rim and C. J. Nelson, J. Appl. Polym. Sci. 42, 1807 (1991).

14. S. K. Koc, A. S. Hockenberger and Q. Wei, Appl. Surf. Sci. 254, 7049 (2008).

15. S. Rebouillat, B. Letellier and B. Steffenino, Int. J. Adhes. Adhes. 19, 303 (1999).

16. D. Beers, R. J. Young, C. L. So, D. J. Sikkema, K. E. Perepelkin and G. Weedon, in: High Performance Fibers, J. S. W. Hearle (Ed.), pp. 93-101. Woodhead Publishing Limited, Cambridge (2001). 